\title{
CpG oligodeoxyribonucleotide 7909 enhances radiosensitivity via downregulating Oct-4 expression in radioresistant lung cancer cells
}

This article was published in the following Dove Press journal:

OncoTargets and Therapy

12 June 2015

Number of times this article has been viewed

\author{
Na Xing' \\ Tiankui Qiao' \\ Xibing Zhuang' \\ Sujuan Yuan' \\ Qi Zhang' \\ Guoxiong $\mathrm{Xu}^{2}$ \\ 'Department of Oncology, ${ }^{2}$ Center \\ Laboratory, Jinshan Hospital, Fudan \\ University, Shanghai, People's Republic \\ of China
}

\begin{abstract}
Radiotherapy is a powerful cure for local advanced non-small cell lung cancer. However, radioresistance and tumor relapse still occur in a high proportion of patients. Octamer-4 (Oct-4), a transcription factor of the POU family, plays a key role in maintaining chemoradioresistant properties and regulating cancer progression. In this study, we demonstrated that Oct-4 expression was significantly increased in radioresistant H460 (H460R) cell line. CpG oligodeoxyribonucleotide (CpG-ODN) 7909 sensitized H460R cells when combined with irradiation treatment. The clonogenic capacity was significantly decreased, and the values of D0 and Dq were lower than those of irradiation alone group. The sensitive enhancement ratio (SER) of D0 was 1.224. This combined treatment led to a dramatic reduction in Oct-4 expression in a dose-dependent manner and also showed increased percentage of cells in the radiosensitive $\mathrm{G} 2 / \mathrm{M}$ phase relative to either treatment alone. These results identified that Oct-4 was involved in radioresistance. CpG-ODN 7909 could enhance radiosensitivity partly through downregulating Oct-4 expression in radioresistant lung cancer cells.
\end{abstract}

Keywords: CpG-ODN, Oct-4, lung cancer, TLR9, radiosensitivity

\section{Introduction}

Lung cancer remains lethal with 5-year survival rate generally lower than 20\%, despite intensive therapeutic efforts. ${ }^{1}$ As a powerful cure for local advanced non-small cell lung cancer (NSCLC), radiotherapy remains largely palliative like most other antitumor therapies due to the adaptive resistance. ${ }^{2}$

Octamer-4 (Oct-4), a transcription factor of the POU family, plays a key role in the maintenance of embryonic stem cell proliferation and pluripotency. ${ }^{3,4}$ Moreover, Oct-4 is also overexpressed in some solid tumors, including breast, bladder, colorectal, renal, and lung cancers, which is associated with the maintenance of cancer stemlike and chemoradioresistant properties..$^{5-12}$ The ectopic expression of Oct-4 controls epithelial-mesenchymal transition and plays an important role in enhancing malignancy and regulating cancer progression. , $^{5,9,13-15}$

Toll-like receptor 9 (TLR9) has been identified to be the receptor conferring CpG reactivity by directly engaging bacterial DNA or synthetic $\mathrm{CpG}$ oligodeoxyribonucleotides (CpG-ODNs) in a $\mathrm{CpG}$ motif-dependent manner. ${ }^{16-20}$ Owing to the immunostimulatory effects, CpG-ODNs targeting TLR9 have been considered as antitumor drugs, as monotherapy or in combination with radiotherapy, chemotherapy, or novel biological treatment. ${ }^{21}$ In previous studies, we demonstrated that CpG-ODNs present radiation-enhancing effects when combined with radiotherapy, which predominantly attributes to the release of cytokines, activation of natural killer cells, and induction of
Correspondence: Tiankui Qiao Department of Oncology, Jinshan Hospital, Fudan University, No 1508 Longhang Road, Shanghai 201508, People's Republic of China

Tel +86 I89 30778786

Email qiaotk@।63.com 
T-cell responses. ${ }^{22-24}$ Because of the overexpression of TLR9 in radiation-resistant human lung adenocarcinoma cell line, CpG-ODNs could even present significant radiosensitizing effects in radioresistant cells in vitro. ${ }^{25}$ However, whether CpG-ODNs affect the cancer stem-like properties of radioresistant lung cancer cells has yet to be elucidated. Accordingly, in the present study, we focus on the effects of CpG-ODNs on Oct-4 expression in radiation-resistant lung cancer cell line.

\section{Materials and methods}

\section{Cell culture}

The human NCI-H460 NSCLC cell line was purchased from Chinese Academy of Sciences (Shanghai, People's Republic of China) and was cultured in RPMI-1640 medium (HyClone, MA,USA) supplemented with $10 \%$ fetal bovine serum at $37^{\circ} \mathrm{C}$ in a humidified atmosphere of $5 \% \mathrm{CO}_{2}$.

\section{Reagents}

CpG-ODN 7909 (5'-TCGTCGTTTTGTCGTTTTGT CGTT-3'-) was selected according to the published reports and purchased from Shanghai Sangon Biological Engineering Technology \& Services Co., Ltd. (Shanghai, People's Republic of China). ${ }^{23,25,26}$ Chloroquine (CQ), a TLR9 inhibitor, was obtained from Sigma-Aldrich Co. (St Louis, MO, USA). CpG-ODN 7909 and CQ were separately resuspended in phosphate buffer saline to $1 \mathrm{mg} / \mathrm{mL}$ stock solutions and maintained at $-20^{\circ} \mathrm{C}$ until use.

\section{Generation of radioresistant cell line}

H460 cells in an exponentially growing phase were irradiated at a dose of 6.37 Gy using a high-energy $6 \mathrm{MV}$ $\mathrm{X}$-ray accelerator delivering $2 \mathrm{~Gy} / \mathrm{min}$ (source-to-surface distance $=100 \mathrm{~cm}$ ). For the generation of radioresistant variant cell line, cells were treated for ten rounds, and this radioresistant cell line was designated as radioresistant $\mathrm{H} 460$ (H460R). ${ }^{25}$

\section{Study design}

H460R cells were divided into six groups and plated in sixwell plates (1.5×105/well): control group, CpG-ODN 7909 group (CpG group), irradiated group (IR group), irradiated + CpG-ODN 7909 group (IR + CpG group), irradiated + CpG-ODN $7909+$ CQ group (IR + CpG + CQ group), and irradiated + CQ group (IR + CQ group). CpG-ODN 7909 was administrated to a final concentration of $20 \mu \mathrm{g} / \mathrm{mL}$ for 24 hours. CQ was used at a final concentration of $10 \mu \mathrm{g} /$ $\mathrm{mL}$ in pretreatment during 1 hour prior to stimulation with CpG-ODN 7909. Then, cells were irradiated with $10 \mathrm{~Gy}$.
Logarithmic growing cells of each group were harvested at 48 hours after irradiation (IR).

\section{Clonogenic survival assay}

Cells that received different treatments were irradiated with graded IR doses ranging from 0 Gy to 8 Gy and then immediately trypsinized and seeded in triplicate in six-well plates (500-3,000 cells per well depending on the expected surviving fraction after $\mathrm{IR}^{27}$ ). After 10 days of incubation, cells were fixed and stained with crystal violet, and colonies $\geq 50$ cells were counted. Clonogenic survival fraction was calculated as (irradiated cell colony numbers/unirradiated cell colony numbers) $\times 100 \%$. Data were analyzed with linear quadratic and single-hit multitarget models.

\section{Western blot analysis}

Western blot analysis was used to determine the expression of Oct-4. The whole cell lysate was prepared using the sodium dodecyl sulfate (SDS) lysis buffer (Beyotime, Nantong, People's Republic of China) with $1 \mathrm{mM}$ phenylmethanesulfonyl fluoride (Beyotime). Protein concentration was determined using Coomassie blue fast staining solution (Beyotime). Protein extracts were separated by $10 \%$ SDS-polyacrylamide gel electrophoresis, transferred to PVDF membranes (EMD Millipore, Billerica, MA, USA), blocked with $5 \%$ nonfat dry milk in tris-buffered saline (TBS) containing $0.1 \%$ Tween-20 (TBS-T) for 2 hours at room temperature, and then incubated with the following primary antibodies at $4{ }^{\circ} \mathrm{C}$ overnight: anti-Oct-4 (Abcam, Cambridge, UK) and anti- $\beta$-actin (Santa Cruz, Dallas, TX, USA). Membranes were washed with TBS-T three times and probed with secondary antibodies for 1 hour at room temperature. Protein bands were visualized using chemiluminescence HRP substrate (EMD Millipore).

\section{Cell cycle assay}

The cell cycle was analyzed by measuring the DNA content stained with propidium iodide in the presence of RNase (BD Biosciences, San Jose, CA, USA) per manufacturer's instructions. The cells were harvested and fixed with $75 \%$ ethanol at $4^{\circ} \mathrm{C}$ overnight and then incubated with $500 \mu \mathrm{L}$ propidium iodide/RNase staining buffer (BD Biosciences) at room temperature for 15 minutes. The percentage of cells at each cell cycle was evaluated by a flow cytometer.

\section{Statistical analysis}

SPSS 21.0 and GraphPad Prism 5.0 were used for data analysis, and the results were presented as mean \pm standard 
deviation. A $P$-value $<0.05$ was considered statistically significant difference.

\section{Results}

\section{Generation of radioresistant lung cancer cells}

The responses to IR between resistant cells and parental cells were analyzed by clonogenic assay. As shown in Figure 1 and Table 1, in the resistant H460R cells, a marked increase in D0 and $N$ values and a broader initial shoulder were observed as compared to parental H460 cells. H460R cells were more resistant to cell death post-IR at each dose tested. These results indicated the acquired radioresistant characteristics of H460R cells.

\section{Elevated expression of Oct-4 in radioresistant lung cancer cells}

Since Oct-4 expression plays an important role in regulating cancer progression and maintaining chemoradioresistant properties, we detected the level of Oct-4 in H460 and H460R cells. As shown in Figure 2, a substantial increase in Oct-4 expression was detected in H460R cells as compared to parental H460 cells, suggesting that Oct-4 was involved in radioresistance, and therefore, the radioresistant lung cancer cells may present more aggressive phenotypes than parental cells.

\section{Effects of CpG-ODN 7909 on expression of Oct-4 post-IR in radioresistant lung cancer cells}

To determine whether CpG-ODN 7909 influences the expression of Oct-4, we treated H460R cells with an increasing

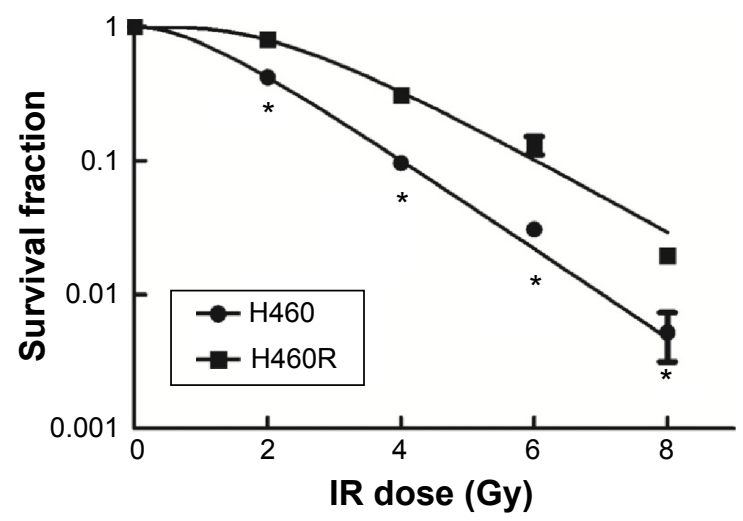

Figure I Analysis of radiosensitivity via clonogenic assay.

Notes: A single-hit multitarget model was used to draw dose-survival curves. The dose-survival curve of H460R cells exhibited a broader initial shoulder (indicating increase in $\mathrm{Dq}$ ) and a smaller slope rate (indicating increase in D0). H460R cells were more resistant to cell death post-IR at each dose tested. $* P<0.05$ vs $\mathrm{H} 460$ cells at each dose tested.

Abbreviations: $\mathrm{H} 460 \mathrm{R}$, radioresistant $\mathrm{H} 460$; IR, irradiation.
Table I Comparison of radiosensitivity between $\mathrm{H} 460$ cells and H460R cells

\begin{tabular}{llll}
\hline Cell line & D0 & Dq & N \\
\hline H460 & 1.299 & 1.056 & 2.254 \\
H460R & 1.569 & 2.481 & 4.862 \\
\hline
\end{tabular}

Notes: The $\mathrm{D} 0, \mathrm{Dq}$, and $N$ values were significantly increased relative to parental $\mathrm{H} 460$ cells, which indicated the acquired radioresistant characteristics of $\mathrm{H} 460 \mathrm{R}$ cells. Abbreviation: $\mathrm{H} 460 \mathrm{R}$, radioresistant $\mathrm{H} 460$.

concentration of CpG-ODN 7909 for 24 hours, exposed them to $10 \mathrm{~Gy}$ IR, and then incubated them for 48 hours. We then detected that, when combined with IR, CpG-ODN 7909 downregulated Oct-4 expression in H460R cells in a dose-dependent manner. Pretreatment of H460R cells with CQ caused a blockade in this reduction (Figure 3). These results indicated that CpG-ODN 7909 could reduce the Oct-4 expression through TLR9 signaling and may further influence the radiosensitivity of radioresistant lung cancer cells.

\section{Effects of CpG-ODN 7909 on the radiosensitivity of radioresistant lung} cancer cells

To assess and characterize the radiation-enhancing effects of CpG-ODN 7909, clonogenic survival analysis was performed. As shown in Figure 4 and Table 2, CpG-ODN 7909 presented significant radiation-enhancing effects. H460R cells treated with CpG-ODN 7909 plus IR exhibited decreased clonogenic capacity and displayed lower values of D0, Dq, and $N$ than those of IR alone group, and the sensitive enhancement ratio (SER) of D0 was1.224. However, after the activation of TLR9 was blocked by CQ, we were unable to detect any radiation-enhancing effects.

\section{Effects of CpG-ODN 7909 on cell cycle regulation after IR in radioresistant lung cancer cells}

To determine if radiosensitizing effects of CpG-ODN 7909 were related to cell cycle regulation, we performed flow cytometric analysis to determine the number of cells in each cell cycle phase. As shown in Figure 5A and B, combined treatment with IR and CpG-ODN 7909 showed an increased percentage of cells in $\mathrm{G} 2 / \mathrm{M}$ phase compared to either treatment alone in H460R cells, which could be blocked by the co-treatment of CQ. The results above suggested that CpG-ODN 7909 allowed H460R cells to produce G2/M arrest through TLR9 signaling.

\section{Discussion}

Lung cancer is the most common diagnosed tumor with a poor prognosis. ${ }^{1}$ Although radiotherapy has been a powerful 
A

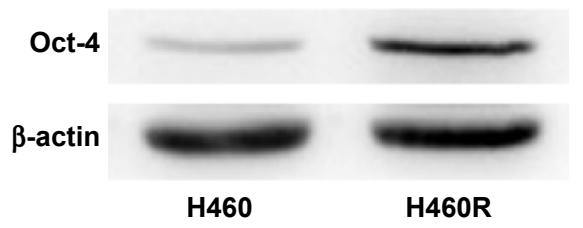

B

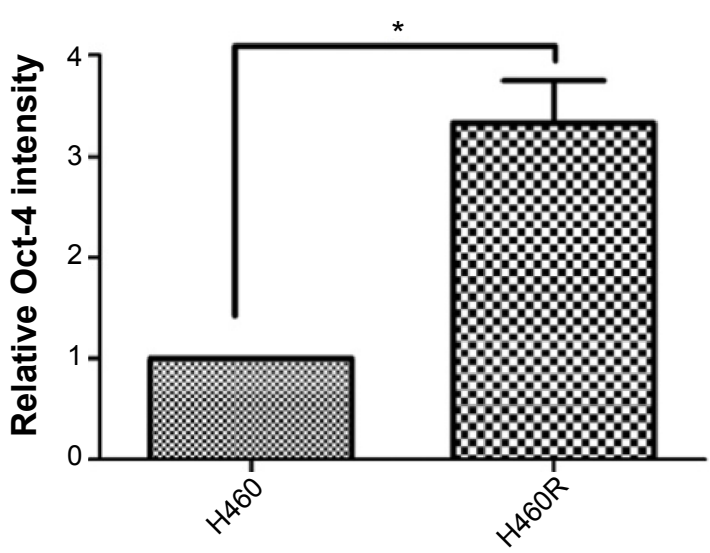

Figure 2 The expression level of Oct- 4 was higher in H460R cells compared with $\mathrm{H} 460$ cells.

Notes: Illustrative Western blots (A) and densitometric analysis (B) demonstrated that Oct-4 expression was significantly increased in $\mathrm{H} 460 \mathrm{R}$ cells relative to parental H460 cells, indicating that Oct-4 was involved in radioresistance. $* P<0.05$ vs $\mathrm{H} 460$ cells.

Abbreviations: Oct-4, octamer-4; H460R, radioresistant $\mathrm{H} 460$.

A

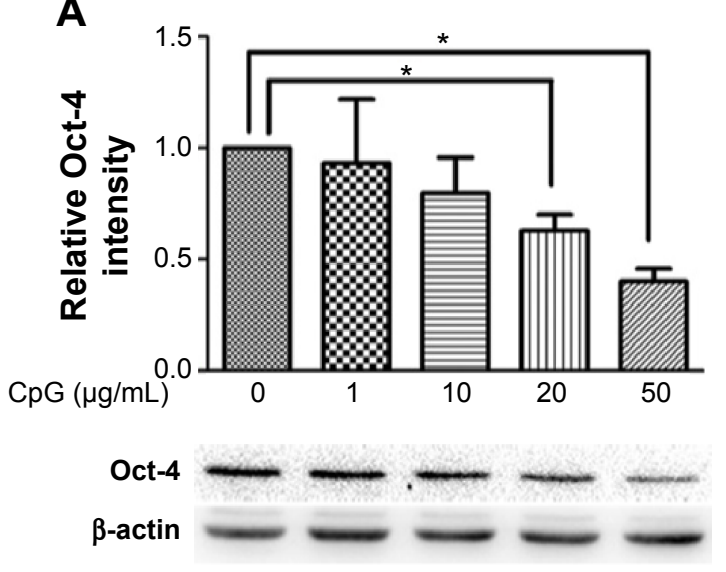

B

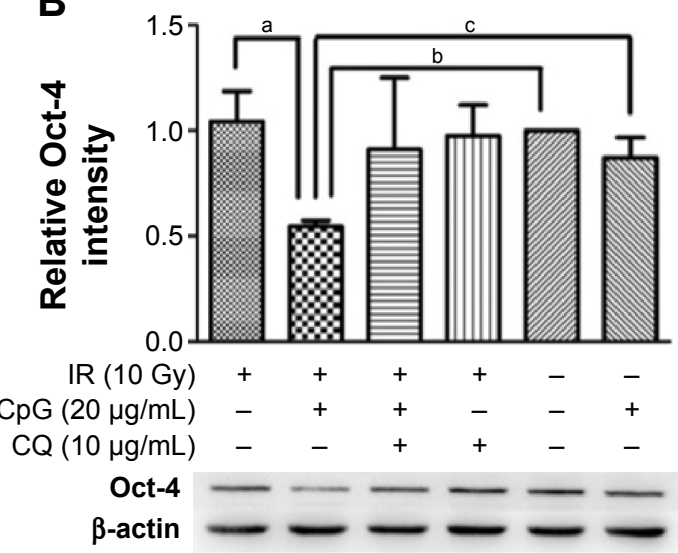

Figure 3 CpG-ODN 7909 downregulated Oct-4 expression in H460R cells when combined with IR.

Notes: Illustrative Western blots and densitometric analysis demonstrated that CpG-ODN 7909 downregulated Oct-4 expression in H460R cells in a dose-dependent manner. This reduction could be blocked by the co-treatment of $C Q$. $* P<0.05$ vs $0 \mu \mathrm{g} / \mathrm{mL}$; (a) $P<0.05$ vs IR group; (b) $P<0.05$ vs control group; and (c) $P<0.05$ vs $C p G$ group. Abbreviations: CPG-ODN 7909, CpG oligodeoxyribonucleotide 7909; Oct-4, octamer-4; H460R, radioresistant H460; IR, irradiation; CQ, chloroquine; IR group, irradiated group.

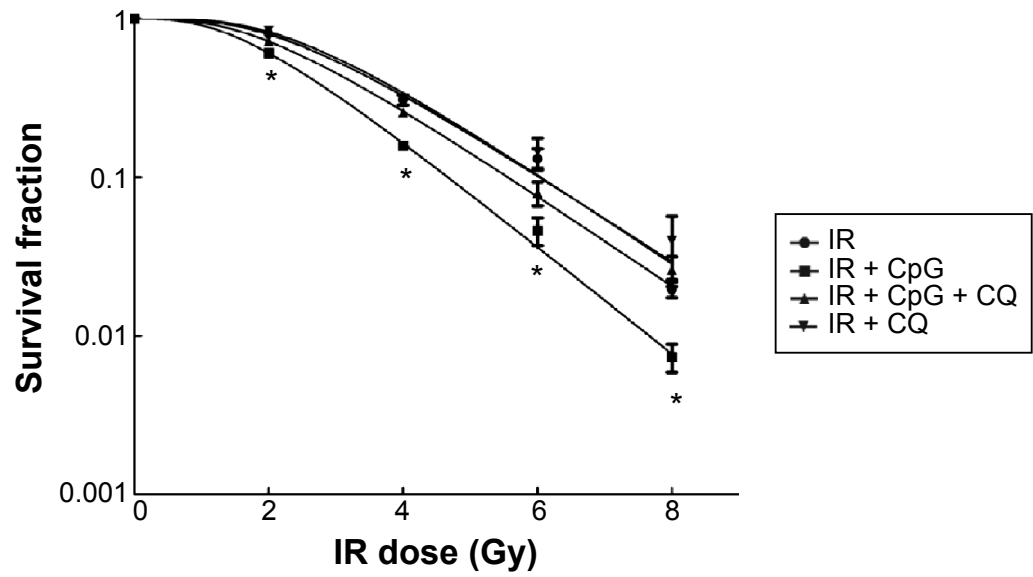

Figure 4 CPG-ODN 7909 presented significant radiation-enhancing effects on H460R cells.

Notes: Combined treatment with IR and CpG-ODN 7909 contributed to further reduction in the survival fractions at each dose tested compared to IR alone $(* P<0.05$ vs IR group at each dose tested). The dose-survival curve of H460R cells treated with CpG-ODN 7909 plus IR exhibited a narrow shoulder (indicating decrease in Dq) and a greater slope rate (indicating decrease in D0), indicating that CpG-ODN 7909 enhanced radiosensitivity when combined with IR treatment. However, the radiation-enhancing effects disappeared after TLR9 activation was blocked by CQ.

Abbreviations: $C_{p}$ G-ODN 7909, CpG oligodeoxyribonucleotide 7909; H460R, radioresistant H460; IR, irradiation; TLR9, toll-like receptor 9; CQ, chloroquine. 
Table 2 Comparison of radiosensitivity of H460R cells treated with IR, CpG-ODN 7909, or CQ

\begin{tabular}{lllll}
\hline Group & D0 & Dq & N & SER \\
\hline IR & 1.569 & $2.48 \mid$ & 4.862 & - \\
IR + CpG & 1.282 & 1.765 & 3.961 & 1.224 \\
IR + CPG + CQ & 1.515 & 2.132 & 4.086 & 1.036 \\
IR + CQ & 1.519 & 2.614 & 5.590 & 1.033 \\
\hline
\end{tabular}

Notes: The D0, Dq, and $N$ values of H460R cells treated with CpG-ODN 7909 plus IR were much lower relative to $\mathrm{H} 460 \mathrm{R}$ cells irradiated alone, indicating the radiosensitization effect of CPG-ODN 7909 plus IR on H460R cells. The SER of D0 was 1.224. However, the radiation-enhancing effects disappeared after TLR9 activation was inhibited by CQ.

Abbreviations: H460R, radioresistant H460; IR, irradiation; CpG-ODN 7909, CPG oligodeoxyribonucleotide 7909; CQ, chloroquine; SER, sensitive enhancement ratio; TLR9, toll-like receptor 9 . cure for locally advanced NSCLC, its efficiency remains limited as tumors develop adaptive response to radiotherapy and become more aggressive, resistant, and invasive. ${ }^{2}$ As a positive regulator of tumor dedifferentiation, Oct-4 plays an important role in maintaining the cancer stem-like and chemoradioresistant properties. ${ }^{10,12}$ In the present study, we found that the resistant $\mathrm{H} 460 \mathrm{R}$ cells showed a significant increase in Oct-4 expression relative to parental cells, which indicated that Oct-4 was associated with the radioresistant phenotype. Thus, targeting Oct-4 might be an effective approach to interfere with the properties of cancer stem-like cells (CSCs) and improve the efficacy of cancer treatments.
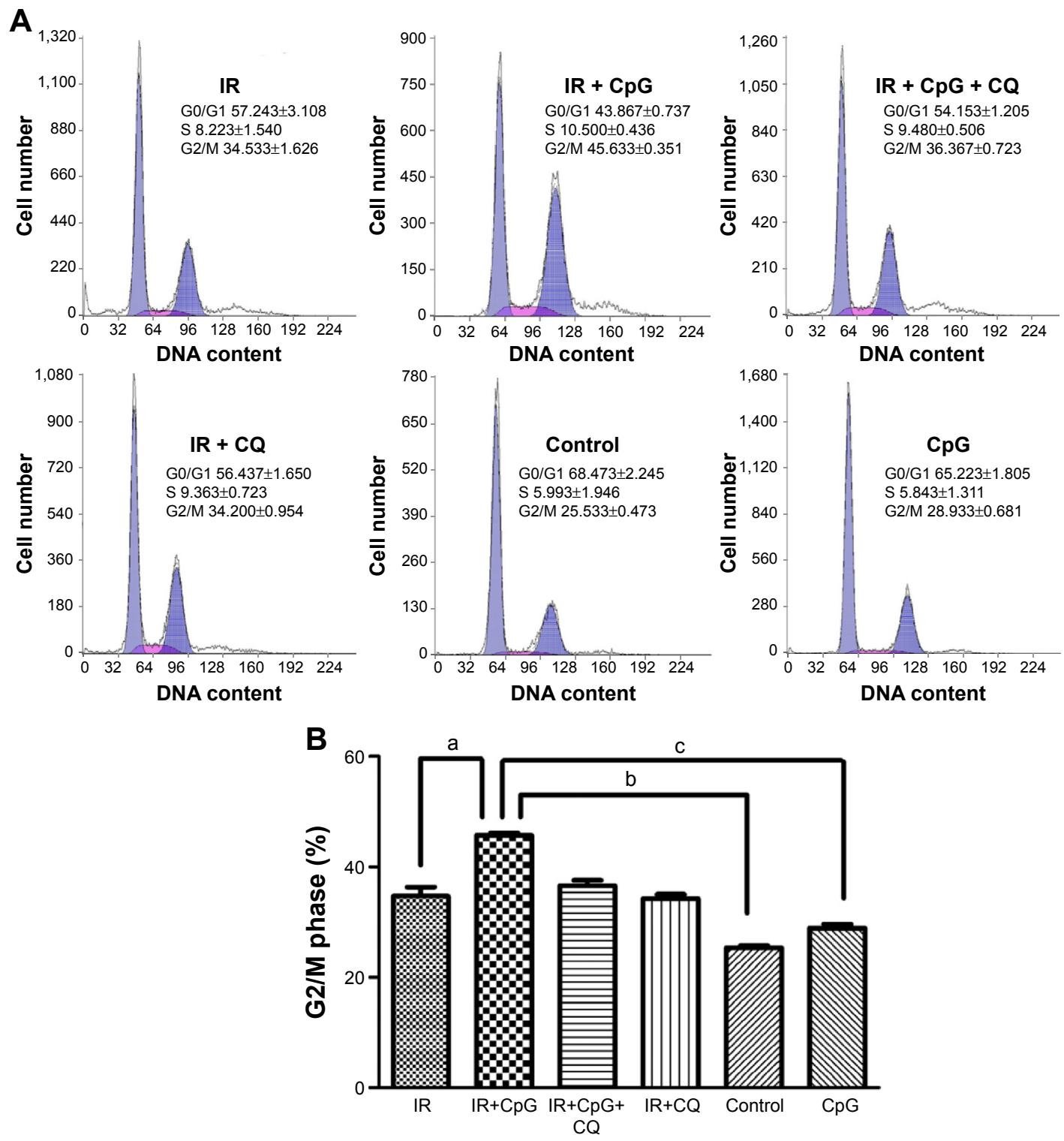

Figure 5 Effect of CpG-ODN 7909 on G2/M phase arrest in H460R cells.

Notes: (A) Flow cytometric analysis of cell cycle distribution. (B) The proportion of cells in the G2/M phase. CPG-ODN 7909 or IR could increase the proportion of cells in the G2/M phase. However, combined treatment showed a much higher percentage in the G2/M phase compared to either treatment alone. This reinforcing effect could be blocked by the co-treatment of CQ, suggesting that $C_{P}$ G-ODN 7909 could prolong cell-cycle arrest in the G2/M phase and enhance radiosensitivity through TLR9 signaling. (a) $P<0.05$ vs IR group; (b) $P<0.05$ vs control group; and (c) $P<0.05$ vs $C p G$ group.

Abbreviations: CPG-ODN 7909, CPG oligodeoxyribonucleotide 7909; H460R, radioresistant H460; IR, irradiation; CQ, chloroquine; TLR9, toll-like receptor 9. 
Due to the immune stimulatory effects, CpG-ODNs have been widely used in cancer therapy, eg, treating oral squamous cell carcinoma, brain tumor, breast cancer, and lung cancer. ${ }^{28-31}$ In our previous studies, we have demonstrated that $\mathrm{CpG}-\mathrm{ODNs}$ could enhance apoptosis, induce prolonged $\mathrm{G} 2 / \mathrm{M}$ arrest, and stimulate TNF- $\alpha$ secretion in human NSCLC cells and thereby enhanced radiosensitivity. As TLR9 was overexpressed in radiation-resistant NSCLC cells, CpG-ODNs could even present significant radiosensitizing effects in radioresistant cells in vitro. ${ }^{23,25}$ All these results urged us to further explore the sensitization mechanism of CpG-ODNs and the influence of CpG-ODNs on Oct-4, as Oct-4 is associated with the radioresistant phenotype.

The ectopic expression of Oct- 4 controls epithelialmesenchymal transition and plays an important role in regulating cancer progression and maintaining the chemoradioresistant properties..$^{5,8,9,13-15} \mathrm{In}$ this study, overexpression of Oct-4 was observed in H460R cells. However, CpG-ODNs induced a dramatic reduction in Oct-4 expression in a dosedependent manner when combined with IR.

TLR9-CpG-DNA interaction is $\mathrm{pH}$ dependent and occurs at the acidic $\mathrm{pH}(6.5-4.5)$ found in endosomes and lysosomes. CQ, a known TLR9 antagonist, is a weak base that can partition into endosomes and perturb endosomal $\mathrm{pH} .{ }^{32,33} \mathrm{CQ}$ has been widely used as the inhibitor for endosomal acidification to inhibit the activation of TLR $9 .{ }^{34-37}$ In the present study, pretreatment of H460R cells with CQ caused a blockade in the reduction of Oct- 4 expression induced by $\mathrm{CpG}-\mathrm{ODNs}$, suggesting that $\mathrm{CpG}$-ODNs could reduce the Oct-4 expression through TLR9 signaling and may therefore present radiosensitizing effects. Cell cycle phase determines cells' relative radiosensitivity, with cells being most sensitive in the G2/M phase. ${ }^{38}$ As shown above, combined treatment with IR and CpG-ODNs led to the accumulation of cells in the G2/M phase. Once the activation of TLR9 was blocked by CQ, the CpG-ODN-induced G2/M arrest was abrogated. These results further demonstrated that $\mathrm{CpG-ODNs}$ sensitized H460R cells when combined with radiotherapy through TLR9 signaling. As shown in Table 2, the SER of D0 was about 1.224.

In summary, Oct-4 was involved in radioresistance. Combined treatment with $\mathrm{CpG}-\mathrm{ODNs}$ and IR presented significant radiosensitizing effects partly through downregulating Oct-4 expression. As cancer stem-like cells phenotype is a dynamic process and can be acquired through Oct-4-mediated dedifferentiation, ${ }^{7,11,12}$ further investigation remains to be performed to study the role of Oct- 4 and the related signaling pathway in radioresistance and to explore the direct effect of CpG-ODNs on CSCs.

\section{Acknowledgment}

This work was supported by the Scientific Fund of the Shanghai Municipal Health Bureau.

\section{Disclosure}

The authors report no conflicts of interest in this work.

\section{References}

1. Allemani C, Weir HK, Carreira H, et al; CONCORD Working Group. Global surveillance of cancer survival 1995-2009: analysis of individual data for 25676887 patients from 279 population-based registries in 67 countries (CONCORD-2). Lancet. 2014;385(9972):977-1010.

2. Kim JJ, Tannock IF. Repopulation of cancer cells during therapy: an important cause of treatment failure. Nat Rev Cancer. 2005;5(7): 516-525.

3. Niwa H, Miyazaki J, Smith AG. Quantitative expression of Oct-3/4 defines differentiation, dedifferentiation or self-renewal of ES cells. Nat Genet. 2000;24(4):372-376.

4. Babaie Y, Herwig R, Greber B, et al. Analysis of Oct4-dependent transcriptional networks regulating self-renewal and pluripotency in human embryonic stem cells. Stem Cells. 2007;25(2):500-510.

5. Liu CG, Lu Y, Wang BB, et al. Clinical implications of stem cell gene Oct-4 expression in breast cancer. Ann Surg. 2011;253(6):1165-1171.

6. Atlasi Y, Mowla SJ, Ziaee SA, Bahrami AR. OCT-4, an embryonic stem cell marker, is highly expressed in bladder cancer. Int $J$ Cancer. 2007;120(7):1598-1602.

7. Wen K, Fu Z, Wu X, Feng J, Chen W, Qian J. Oct-4 is required for an antiapoptotic behavior of chemoresistant colorectal cancer cells enriched for cancer stem cells: effects associated with STAT3/Survivin. Cancer Lett. 2013;333(1):56-65.

8. Kim K, Ro JY, Kim S, Cho YM. Expression of stem-cell markers OCT-4 and CD133: important prognostic factors in papillary renal cell carcinoma. Hum Pathol. 2012;43(12):2109-2116.

9. Chen Z, Wang T, Cai L, et al. Clinicopathological significance of nonsmall cell lung cancer with high prevalence of Oct-4 tumor cells. $J$ Exp Clin Cancer Res. 2012;31:10.

10. Chen YC, Hsu HS, Chen YW, et al. Oct-4 expression maintained cancer stem-like properties in lung cancer-derived CD133-positive cells. PLoS One. 2008;3(7):e2637.

11. Hu T, Liu S, Breiter DR, Wang F, Tang Y, Sun S. Octamer 4 small interfering RNA results in cancer stem cell-like cell apoptosis. Cancer Res. 2008;68(16):6533-6540.

12. Kumar SM, Liu S, Lu H, et al. Acquired cancer stem cell phenotypes through Oct4-mediated dedifferentiation. Oncogene. 2012;31(47): 4898-4911.

13. Wang D, Lu P, Zhang H, et al. Oct-4 and Nanog promote the epithelial-mesenchymal transition of breast cancer stem cells and are associated with poor prognosis in breast cancer patients. Oncotarget. 2014;5(21):10803-10815.

14. Chiou SH, Wang ML, Chou YT, et al. Coexpression of Oct4 and Nanog enhances malignancy in lung adenocarcinoma by inducing cancer stem cell-like properties and epithelial-mesenchymal transdifferentiation. Cancer Res. 2010;70(24):10433-10444.

15. Huang P, Chen J, Wang L, et al. Implications of transcriptional factor, OCT-4, in human bladder malignancy and tumor recurrence. Med Oncol. 2012;29(2):829-834.

16. Vollmer J, Krieg AM. Immunotherapeutic applications of CpG oligodeoxynucleotide TLR9 agonists. Adv Drug Deliv Rev. 2009;61(3): 195-204.

17. Hemmi H, Takeuchi O, Kawai T, et al. A toll-like receptor recognizes bacterial DNA. Nature. 2000;408(6813):740-745.

18. Bauer S, Kirschning CJ, Häcker H, et al. Human TLR9 confers responsiveness to bacterial DNA via species-specific $\mathrm{CpG}$ motif recognition. Proc Natl Acad Sci U S A. 2001;98(16):9237-9242. 
19. Cornélie S, Hoebeke J, Schacht AM, et al. Direct evidence that tolllike receptor 9 (TLR9) functionally binds plasmid DNA by specific cytosine-phosphate-guanine motif recognition. J Biol Chem. 2004; 279(15):15124-15129.

20. Latz E, Schoenemeyer A, Visintin A, et al. TLR9 signals after translocating from the ER to CpG DNA in the lysosome. Nat Immunol. 2004; 5(2):190-198.

21. Holtick U, Scheulen ME, von Bergwelt-Baildon MS, Weihrauch MR. Toll-like receptor 9 agonists as cancer therapeutics. Expert Opin Investig Drugs. 2011;20(3):361-372.

22. Li R, Song Y, Chen W. Enhancing radiosensitivity of human pulmonary adenocarcinoma cell line A549 by CpG ODN1826. Cancer Biother Radiopharm. 2011;26(1):69-76.

23. Zha L, Qiao T, Yuan S, Lei L. Enhancement of radiosensitivity by CpGoligodeoxyribonucleotide-7909 in human non-small cell lung cancer A549 cells. Cancer Biother Radiopharm. 2010;25(2):165-170.

24. Yuan S, Qiao T, Chen W. CpG oligodeoxynucleotide 1826 enhances the Lewis lung cancer response to radiotherapy in murine tumor. Cancer Biother Radiopharm. 2011;26(2):203-208.

25. Yan L, Xu G, Qiao T, Chen W, Yuan S, Li X. CpG-ODN 7909 increases radiation sensitivity of radiation-resistant human lung adenocarcinoma cell line by overexpression of toll-like receptor 9. Cancer Biother Radiopharm. 2013;28(7):559-564.

26. Witzig TE, Wiseman GA, Maurer MJ, et al. A phase I trial of immunostimulatory CpG 7909 oligodeoxynucleotide and 90 yttrium ibritumomab tiuxetan radioimmunotherapy for relapsed B-cell non-Hodgkin lymphoma. Am J Hematol. 2013;88(7):589-593.

27. Short SC, Giampieri S, Worku M, et al. Rad51 inhibition is an effective means of targeting DNA repair in glioma models and CD133+ tumorderived cells. Neuro Oncol. 2011;13(5):487-499.

28. Ruan M, Thorn K, Liu S, et al. The secretion of IL-6 by CpG-ODNtreated cancer cells promotes $\mathrm{T}$-cell immune responses partly through the TLR-9/AP-1 pathway in oral squamous cell carcinoma. Int J Oncol. 2014;44(6):2103-2110.
29. Jarry U, Donnou S, Vincent M, et al. Treg depletion followed by intracerebral CpG-ODN injection induce brain tumor rejection. J Neuroimmunol. 2014;267(1-2):35-42.

30. Xia Y, Gupta GK, Castano AP, Mroz P, Avci P, Hamblin MR. CpG oligodeoxynucleotide as immune adjuvant enhances photodynamic therapy response in murine metastatic breast cancer. J Biophotonics. 2014; 7(11-12):897-905.

31. Sorrentino R, Morello S, Forte G, et al. B cells contribute to the antitumor activity of $\mathrm{CpG}$-oligodeoxynucleotide in a mouse model of metastatic lung carcinoma. Am J Respir Crit Care Med. 2011;183(10): 1369-1379.

32. Mellman I, Fuchs R, Helenius A. Acidification of the endocytic and exocytic pathways. Annu Rev Biochem. 1986;55:663-700.

33. Rutz M, Metzger J, Gellert T, et al. Toll-like receptor 9 binds singlestranded CpG-DNA in a sequence- and pH-dependent manner. Eur $J$ Immunol. 2004;34(9):2541-2550.

34. Fiola S, Gosselin D, Takada K, Gosselin J. TLR9 contributes to the recognition of EBV by primary monocytes and plasmacytoid dendritic cells. J Immunol. 2010;185(6):3620-3631.

35. Kuznik A, Bencina M, Svajger U, Jeras M, Rozman B, Jerala R. Mechanism of endosomal TLR inhibition by antimalarial drugs and imidazoquinolines. J Immunol. 2011;186(8):4794-4804.

36. Tillack K, Breiden P, Martin R, Sospedra M. T lymphocyte priming by neutrophil extracellular traps links innate and adaptive immune responses. J Immunol. 2012;188(7):3150-3159.

37. Sanjuan MA, Rao N, Lai KT, et al. CpG-induced tyrosine phosphorylation occurs via a TLR9-independent mechanism and is required for cytokine secretion. J Cell Biol. 2006;172(7):1057-1068.

38. Pawlik TM, Keyomarsi K. Role of cell cycle in mediating sensitivity to radiotherapy. Int J Radiat Oncol Biol Phys. 2004;59(4):928-942.
OncoTargets and Therapy

\section{Publish your work in this journal}

OncoTargets and Therapy is an international, peer-reviewed, open access journal focusing on the pathological basis of all cancers, potential targets for therapy and treatment protocols employed to improve the management of cancer patients. The journal also focuses on the impact of management programs and new therapeutic agents and protocols on

\section{Dovepress}

patient perspectives such as quality of life, adherence and satisfaction. The manuscript management system is completely online and includes a very quick and fair peer-review system, which is all easy to use. Visit http://www.dovepress.com/testimonials.php to read real quotes from published authors. 